\title{
Effect of Thickness on Fatigue Crack Propagation in Injection Molded Short Carbon Fiber Reinforced Plastics
}

\author{
Kenichi Shimizu ${ }^{1, *}$, Yuya Hasegawa ${ }^{2}$ and Keisuke Tanaka ${ }^{3}$ \\ ${ }^{1}$ Department of Mechanical Engineering, Meijo University, Nagoya, Japan \\ ${ }^{2}$ Graduate School, Department of Mechanical Engineering, Meijo University, Nagoya, Japan \\ ${ }^{3}$ Department of Research, Nagoya Industrial Science Research Institute, Nagoya, Japan
}

\begin{abstract}
The influence of plate thickness on the fatigue crack propagation behavior was studied by using center-notched specimens which were cut from injection-molded plates of short carbon-fiber reinforced polyphenylene sulfide (PPS) at two fiber angles relative to the molding flow direction (MFD), i. e. $\theta=0$ deg. (MD), 90 deg. (TD). The short carbon-fiber reinforced plastics (SCFRP) plates have three-layer structure where the fiber orientation is parallel to MFD in the shell layer and is nearly perpendicular in the core layer. The fraction of the core layer increases with increase in the plate thickness. In the relation between the crack propagation rate, $d a / d N$, and stress intensity factor, $\Delta K, d a / d N$ increases with increase in thickness for MD specimen. Conversely, $d a / d N$ decreases for TD specimen. The crack opening displacement along the crack was measured by using the digital image correlation (DIC) method. The measured crack opening displacement become larger with increase in the plate thickness for MD specimens. Contrary, measured values become smaller with increase in the plate thickness for TD specimen. The crack-tip-opening radius, $\Delta \rho$, was estimated from the parabolic approximation of the crack opening displacement distribution near the crack tip. The relationships between $d a / d N$ and $\Delta \rho$ for all specimens tend to merge into a unique relationship.
\end{abstract}

\section{Introduction}

Short-fiber reinforced plastics (SFRP) are expected to be used more widely in order to reduce the weight of vehicles such as automobiles. The injection molding process makes the production of SFRP components more economical and at higher rates. The mechanical properties of SFRP components are very much anisotropic, depending on the fiber orientation produced by injection molding. Since the applications of SFRP in fatigue-sensitive components are steadily increasing in automobile industries, the anisotropic fatigue properties should be assessed in relation to the fiber orientation.

The molded SCFRP plates have three-layer structure where the fiber orientation in the shell layer is parallel to molding flow direction (MFD) and that of the core layer is nearly perpendicular to MFD. The fiber orientation produced by injection molding has a big influence on the propagation rate and path of fatigue cracks [1-8]. Because the crack propagation rate perpendicular to aligned fibers is much slower than that parallel to fibers, the crack propagation rate is different between in the shell layer and in the core layer.

The thickness of the shell layer does not increase even if the plate thickness increases, i. e. the fraction of the core layer increases with increase in the plate thickness. Therefore, it is necessary to investigate the effect of thickness of the molded SCFRP plate on the crack propagation behavior.

In this study, the influence of plate thickness on the fatigue crack propagation behavior was discussed by using center-notched specimens which were cut from injection-molded plates of short carbon-fiber reinforced polyphenylene sulfide (PPS) at two fiber angles relative to the molding flow direction (MFD), i. e. $\theta=0$ deg. (MD), 90 deg. (TD). The thickness of plates was 1, 2 and $3 \mathrm{~mm}$. And the crack opening displacement along the crack was measured by using the digital image correlation (DIC) method. The crack-tip-opening radius, $\Delta \rho$, was estimated from the parabolic approximation of the crack opening displacement distribution near the crack tip and the crack propagation rate in each thickness was estimated by using $\Delta \rho$.

\section{Experimental procedure}

\subsection{Specimens}

The experimental material is semi-crystalline brittle thermoplastics, PPS, reinforced with carbon fibers. The amount of fiber content was $30 \mathrm{wt} \%$. Fatigue specimens were cut from an injection-molded plate (IMP) with the in-plane dimensions of $80 \times 80 \mathrm{~mm}$ and the thickness of 1,2 and $3 \mathrm{~mm}$. IMP have a three-layer structure where 
two shell layers sandwich the core layer as shown schematically in Fig. 1(a). Figure 1(b) shows the cross section "A" observed by the optical microscope in IMP with the thickness of $1 \mathrm{~mm}$ (IMP1), 2mm (IMP2) and $3 \mathrm{~mm}$ (IMP3). In the shell layer, short fibers are parallel to the molding direction. In the core layer, fibers are transverse to the molding direction. The thickness of the shell layer does not increase even if the plate thickness increases, i. e. the fraction of the core layer increases with increase in the plate thickness. Table 1 shows the fraction of shell layer and core layer, $f_{\mathrm{s}}$, and $f_{\mathrm{c}}$, respectively.

Figure 2 shows the shape of test specimens which has a center notch of whole length $6 \mathrm{~mm}$, the length of 80 $\mathrm{mm}$, and the width of $20 \mathrm{~mm}$. The region of length 15 $\mathrm{mm}$ was used for chucking to the testing machines through aluminum tabs. A fatigue crack was started from the initial notch, and extended in the direction as shown in Fig.1. The angle between the molding direction and the longitudinal direction of specimens was set to be 0 deg. (MD) and 90 deg. (TD).

The core layer may influence the crack propagation behavior of IMP. To evaluate its influence, specimens made of only skin layer were manufactured by thinning the $1 \mathrm{~mm}$ thickness to $0.4 \mathrm{~mm}$ from the one side of plates only for cases of MD, TD. The layer was carefully removed by milling machines. The surface of the machined layer was polished by emery paper to eliminate damages due to machining and the single edge notch of length $2 \mathrm{~mm}$ was machined. In the following, specimens made of skin-layer are called SLP.

Experimental values of anisotropic elastic constants of IMP and SLP are summarized in Table 2, where suffix 1 indicates the molding direction and 2 the perpendicular direction. The $E_{1}$ value decreases with increase in thickness. Conversely, the $E_{2}$ increases with increase in thickness. In short, the core layer of IMP reduced $E_{1}$ value and increased $E_{2}$ value.

Table 1. Fraction of shell layer and core layer to the thickness of the molded plate.

\begin{tabular}{c|cc}
\hline Specimen & $\begin{array}{c}\text { Fraction of shell layer } \\
f_{\mathrm{s}}(\%)\end{array}$ & $\begin{array}{c}\text { Fraction of core layer } \\
f_{\mathrm{c}}(\%)\end{array}$ \\
\hline IMP1 & $86(=43 \times 2)$ & 14 \\
IMP2 & $56(=28 \times 2)$ & 44 \\
IMP3 & $40(=20 \times 2)$ & 60 \\
\hline
\end{tabular}

Table 2. Elastic constants of injection-molded plate (IMP) and shell layer plate (SLP).

\begin{tabular}{c|c|cccc}
\hline Specimen & $\begin{array}{c}E_{1} \\
(\mathrm{GPa})\end{array}$ & $v_{12}$ & $\begin{array}{c}E_{2} \\
(\mathrm{GPa})\end{array}$ & $v_{21}$ & $\begin{array}{c}G_{12} \\
(\mathrm{GPa})\end{array}$ \\
\hline SLP & 29.76 & 0.418 & 8.94 & 0.131 & 4.36 \\
IMP1 & 28.01 & 0.379 & 12.63 & 0.174 & 4.73 \\
IMP2 & 21.79 & 0.264 & 15.58 & 0.199 & 4.12 \\
IMP3 & 14.97 & 0.196 & 18.68 & 0.229 & 3.72 \\
\hline
\end{tabular}

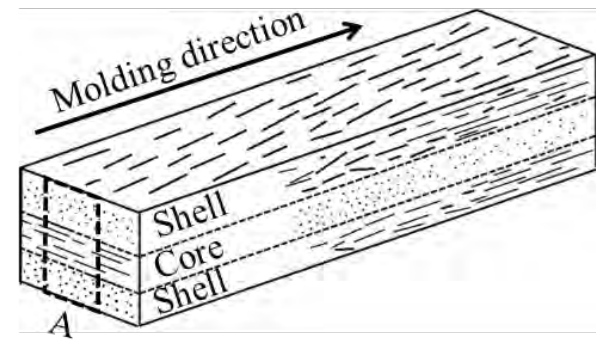

(a) Cross section observed by optical microscope

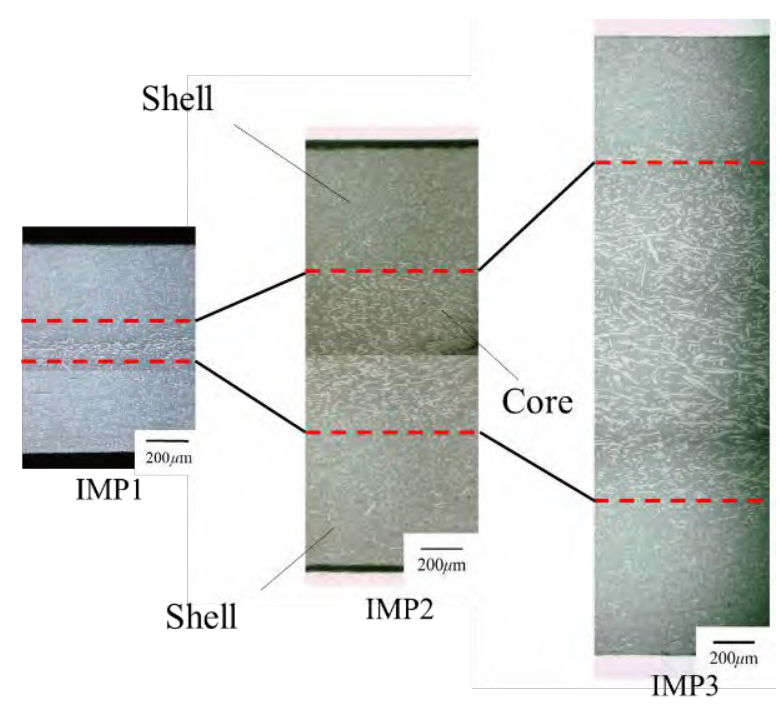

(b) Cross section observed by optical microscope

Fig. 1. Three-layer structure in injection-molded plate (IMP).

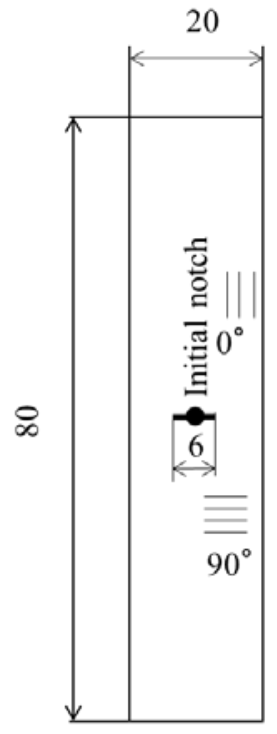

(a) IMP.

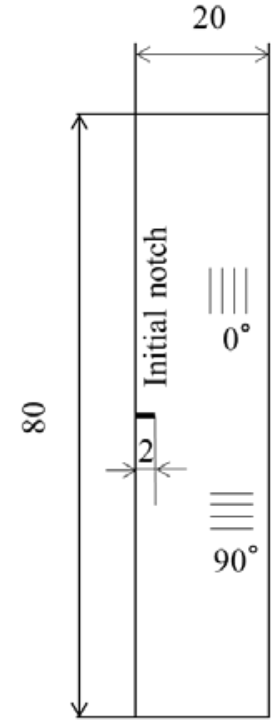

(b) SLP.
Fig. 2. Specimen (in mm). 


\subsection{Fatigue crack propagation tests}

Fatigue crack propagation tests were performed with a tension-compression electro-servo-hydraulic testing machine. Fatigue testing was done in air at room temperature under load-controlled conditions with the stress ratio $R$ of 0.1 . The waveform of the cyclic load was triangular and the frequency was $4 \mathrm{~Hz}$. The crack length was measured with a video microscope. As the macroscopic fatigue crack propagation path was perpendicular to the loading axis, the crack length projected on the plane perpendicular to the loading axis was measured. The crack propagation rate, $d a / d N$, is calculated from crack lengths $a$ vs number of cycles by the secant method.

\subsection{Fracture mechanics parameters}

The energy release rate of crack propagation was calculated by the method of a modified crack closure integral (MCCI) of FEM $[9,10]$. For the case of crack growth under mode I in TD specimen, the energy release rate is converted to the stress intensity factor by using $[11,12]$

$$
\begin{gathered}
G=H K^{2} \\
H=\sqrt{\frac{1}{2 E_{1} E_{2}}}\left[\sqrt{\frac{E_{1}}{E_{2}}}+\frac{-2\left(v_{12} / E_{1}\right)+\left(1 / G_{12}\right)}{2\left(1 / E_{1}\right)}\right]^{1 / 2}
\end{gathered}
$$

For the MD specimen, $H$ is calculated by replacing subscript, 1 and 2 in eq. (1). The $H$ values for MD and TD of IMP and SLP are summarized in Table 3. For isotropic materials, we have $1 / H$ equal to Young's modulus $E$ and

$$
G=K^{2} / E
$$

The stress intensity factor for mode I, $K_{\mathrm{I}}$, is expressed as

$$
K=\sigma \sqrt{\pi a} \cdot F(a / W)
$$

where $F(a / W)$ is a correction factor for the stress intensity factor.

Table 3. Stiffness parameter for MD and TD of IMP and SLP.

\begin{tabular}{c|cc}
\hline Specimen & $\begin{array}{c}\text { MD } \\
\left(\times 10^{-3} / \mathrm{GPa}\right)\end{array}$ & $\begin{array}{c}\text { TD } \\
\left(\times 10^{-3} / \mathrm{GPa}\right)\end{array}$ \\
\hline SLP & 52.2 & 95.2 \\
IMP1 & 50.9 & 75.9 \\
IMP2 & 61.3 & 72.4 \\
IMP3 & 77.8 & 69.6 \\
\hline
\end{tabular}

\subsection{Measurement of crack displacement distribution}

opening

A pair of areas of $18 \times 18 \mu \mathrm{m}$ rectangle (subset) was defined longitudinally at a gage length of $60 \mu \mathrm{m}$ containing a crack line in the image obtained from the digital microscope. Each subset was spaced at $9 \mu \mathrm{m}$ intervals near the crack tip and $110 \mu \mathrm{m}$ other than that along a crack. From images around the fatigue crack under the maximum loading and the minimum loading, the displacements of each subset were evaluated by using digital image correlation method and the crack opening displacement distribution along the fatigue crack was obtained.

\section{Experimental results and discussion}

\subsection{Fatigue crack propagation}

Figure 3 shows optical micrographs of a fatigue crack in MD specimen. The crack propagation is blocked by fibers and the crack path is microscopically zigzag shaped and macroscopically straight perpendicular to the loading direction. But there is no clear difference depending on the thickness. Figure 4 shows a fatigue crack in TD specimen. The zigzag of the crack propagation path is smaller than that in MD specimen for each thickness of the plate.

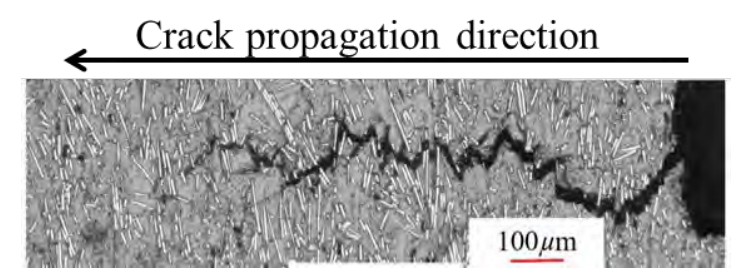

(a) SLP.

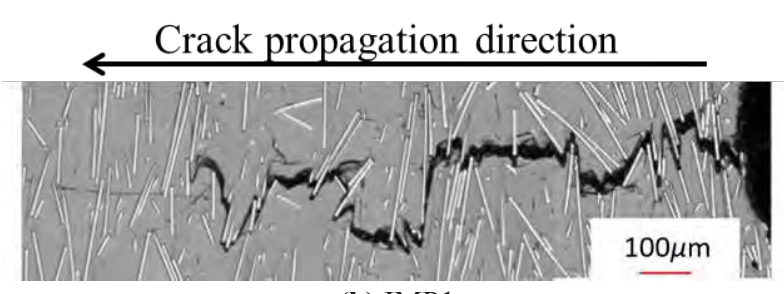

(b) IMP1.

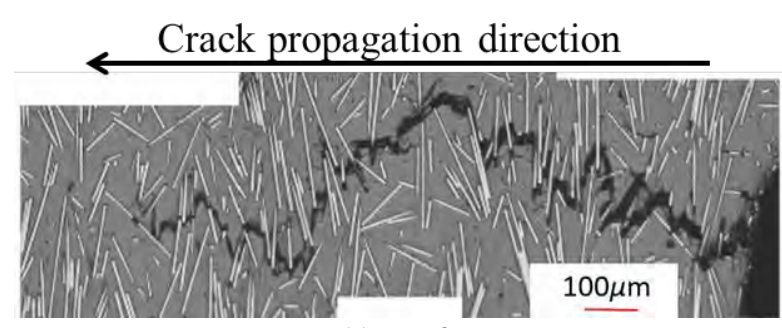

(c) IMP2.

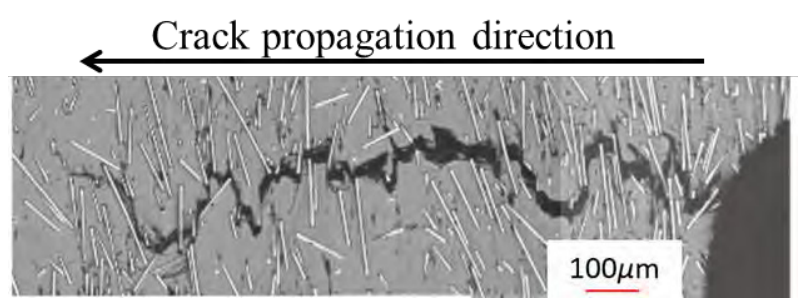

(d) IMP3.

Fig. 3. Optical micrographs of a fatigue crack in MD specimen. 


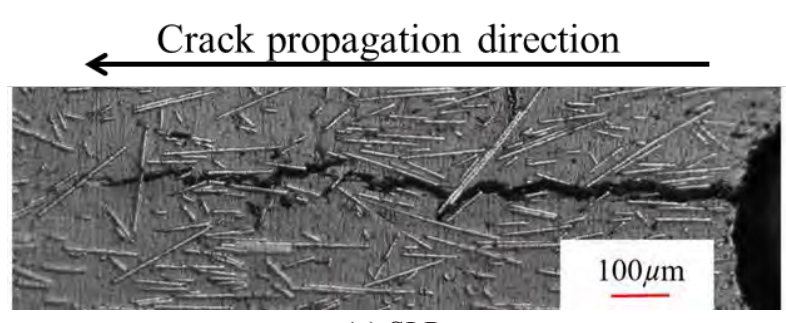

(a) SLP.

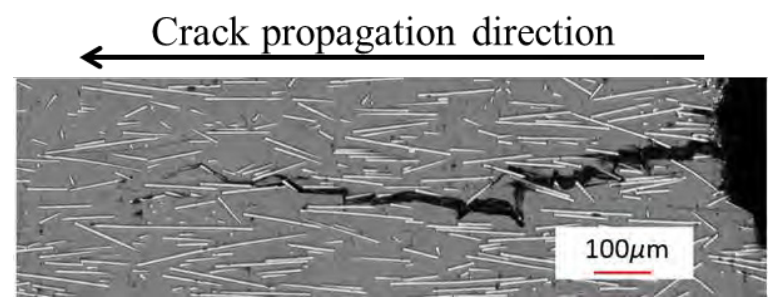

(b) IMP1.

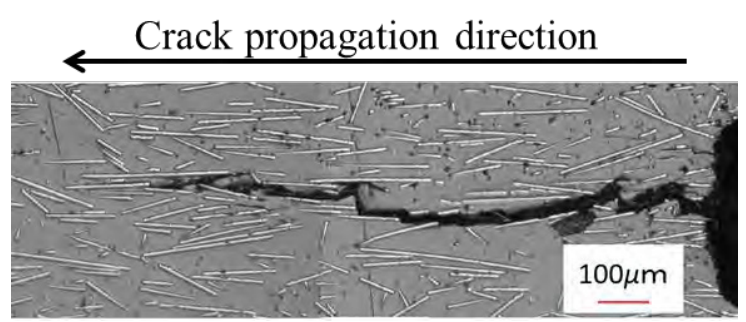

(c) IMP2.

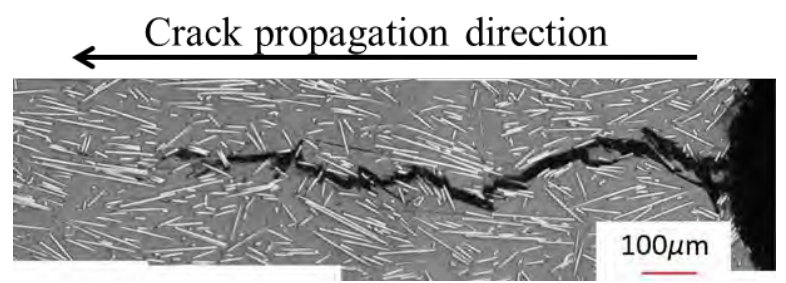

(d) IMP3.

Fig. 4. Optical micrographs of a fatigue crack in TD specimen.

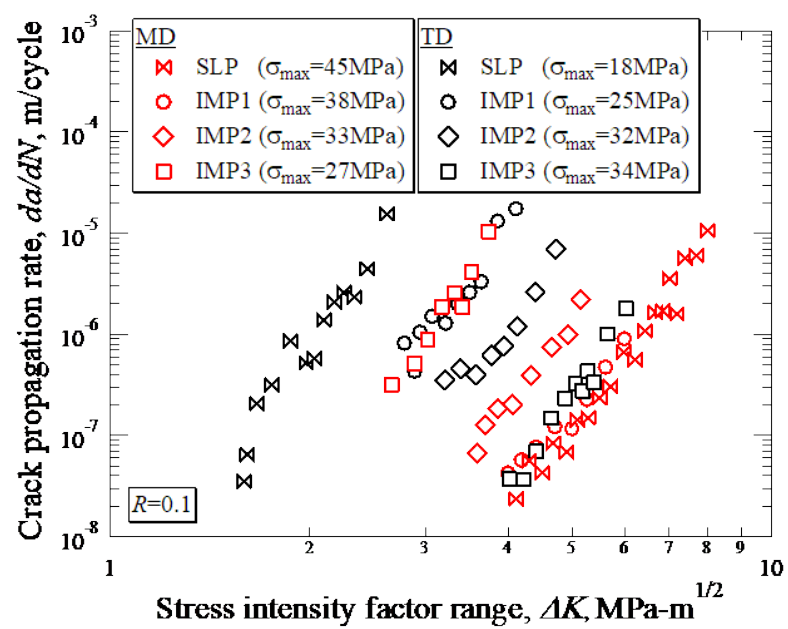

Fig. 5. Relation between crack propagation rate and stress intensity factor range.
The fatigue crack propagation rate, $d a / d N$, is plotted against the range of stress intensity factor, $\Delta K$, in Fig. 5. There is a linear relation between $d a / d N$ and $\Delta K$ in log$\log$ diagram. In the relation between the crack propagation rate, $d a / d N$, and stress intensity factor, $\Delta K$, $d a / d N$ increases with increase in thickness for MD specimen including SLP. The resistance to crack propagation is improved by fiber reinforcement, and fibers aligned perpendicular to the crack growth direction block more severely crack propagation. Then, $d a / d N$ increases with increase in the thickness for the MD specimen because the ratio of shell layer decreases. Conversely, $d a / d N$ decreases with increase in thickness for TD specimen as the ratio of shell layer decreases Because the fiber is parallel to the crack propagation direction in the shell layer, the crack propagates faster than in the core layer for TD specimen.

\subsection{Crack opening displacement}

Figures 6 and 7 show the crack opening displacement obtained from the MD and TD specimen, respectively. Lines are the values calculated by Finite Element Method (FEM) analysis. Two-dimensional isoparametric four-node rectangular elements were used under the condition of plane stress. The orthotropic elastic constants used for FEM is given in Table 1. The influence of layered structure was ignored.

Measured values are considerably smaller than analytical values in SLP of MD specimen as shown in Fig. 6(a). Crack closure is occurred by the rough surface contact or the fiber bridging at the crack tip. Then measured values become larger with increase in the plate thickness as indicated in Fig. 6(b) (d). On the other hand, measured values are little larger than analytical values in SLP of TD specimen as shown in Fig. 7(a). There is a possibility that the crack opening was promoted by delamination between the fiber and the matrix. Contrary to the MD specimen, measured values become smaller with increase in the plate thickness as indicated in Fig. 7(b) (d).

\subsection{Crack-tip-opening radius}

For isotropic materials, the stress intensity factor divided by Young's modulus, $\Delta K / E$, is often used to correlate the crack propagation data for different materials. This parameter can be interpreted to represent the range of the radius of crack-tip opening during one cycle [13]. The crack-tip opening radius is given by

$$
\rho=(4 / \pi)(K / E)^{2}=(4 / \pi) G / E
$$

The range of the crack-tip-opening radius is

$$
\begin{aligned}
\Delta \rho= & \rho_{\max }-\rho_{\min }=(4 / \pi E) \cdot\left(G_{\max }-G_{\min }\right)=(4 / \pi E) \cdot \Delta G \\
& =(4 / \pi) \cdot[(1+R) /(1-R)] \cdot(\Delta K / E)^{2}
\end{aligned}
$$

and the range of the energy release rate is defined by

$$
\Delta G=G_{\max }-G_{\min }
$$

where $G_{\max }$ and $G_{\min }$ are the energy release rate at the maximum and minimum stresses. For orthotropic 
materials, the crack-tip-opening radius is expressed as [12]

$$
\rho=(4 / \pi) H G
$$

so the range of crack-tip-opening radius is given by

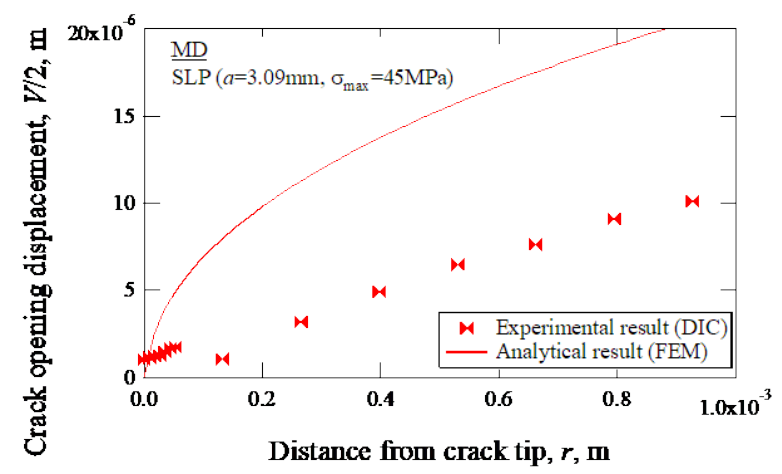

(a) SLP.

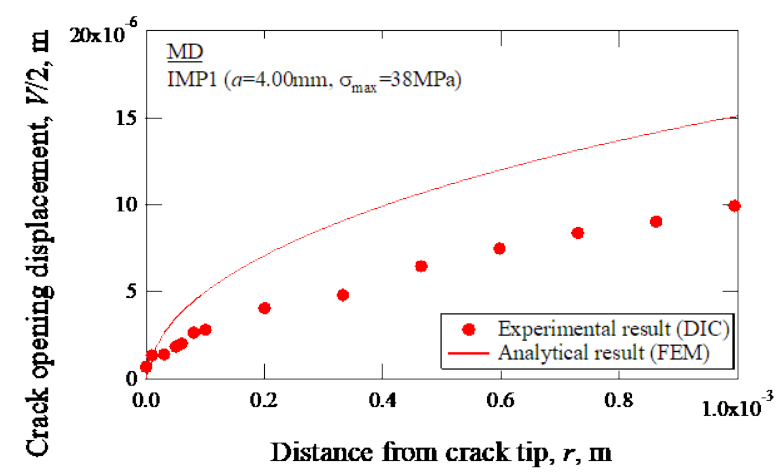

(b) IMP1.

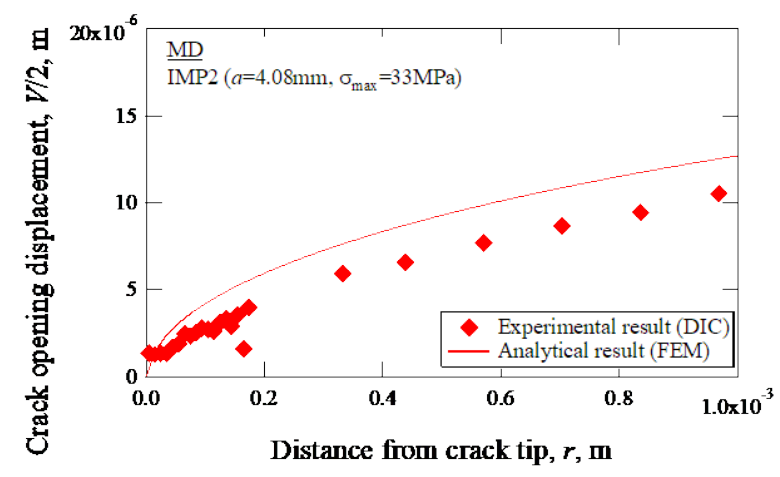

(c) IMP2.

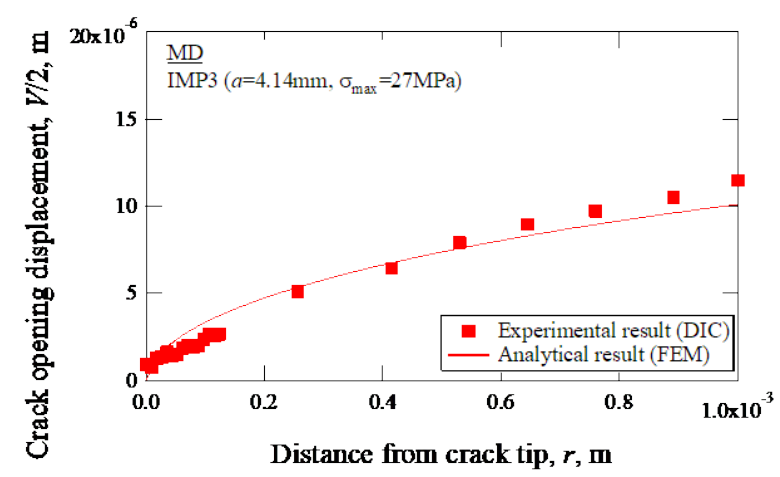

(d) IMP3.

Fig. 6. Crack opening displacement along a fatigue crack in MD specimen.

$$
\Delta \rho=\rho_{\max }-\rho_{\min }=(4 / \pi) H \Delta G
$$

and the range of the energy release rate is defined by

$$
\begin{aligned}
\Delta G= & G_{\max }-G_{\min }=H\left(K_{\max }^{2}-K_{\min }^{2}\right) \\
& =H[(1+R) /(1-R)](\Delta K)^{2}
\end{aligned}
$$

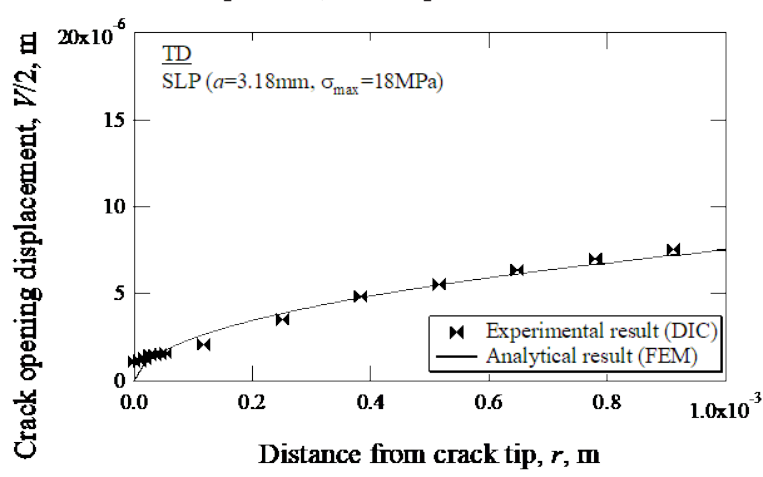

(a) SLP.

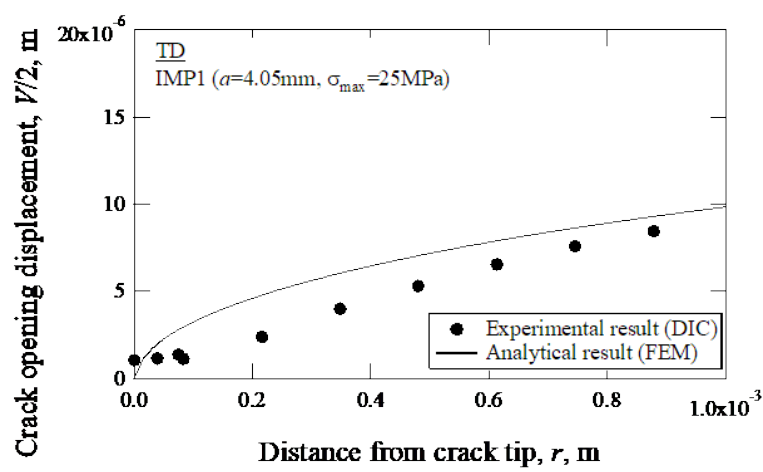

(b) IMP1.

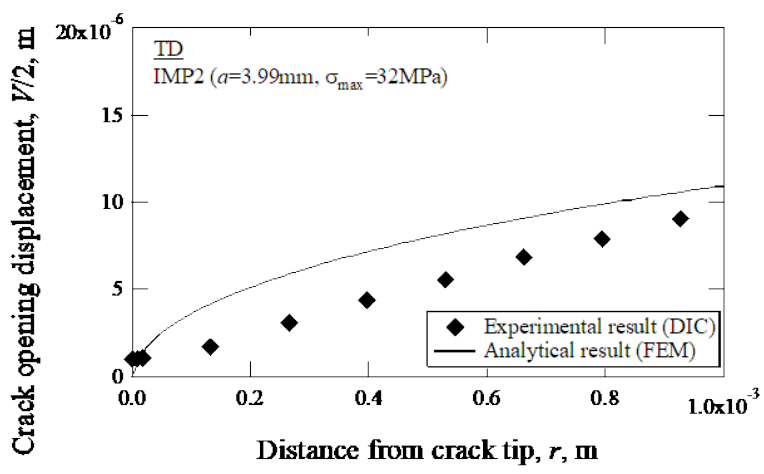

(c) IMP2.

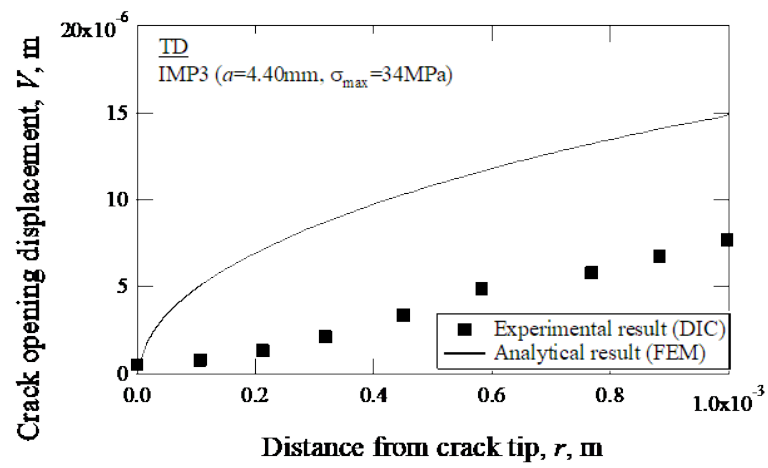

(d) IMP3.

Fig. 7. Crack opening displacement along a fatigue crack in TD specimen. 


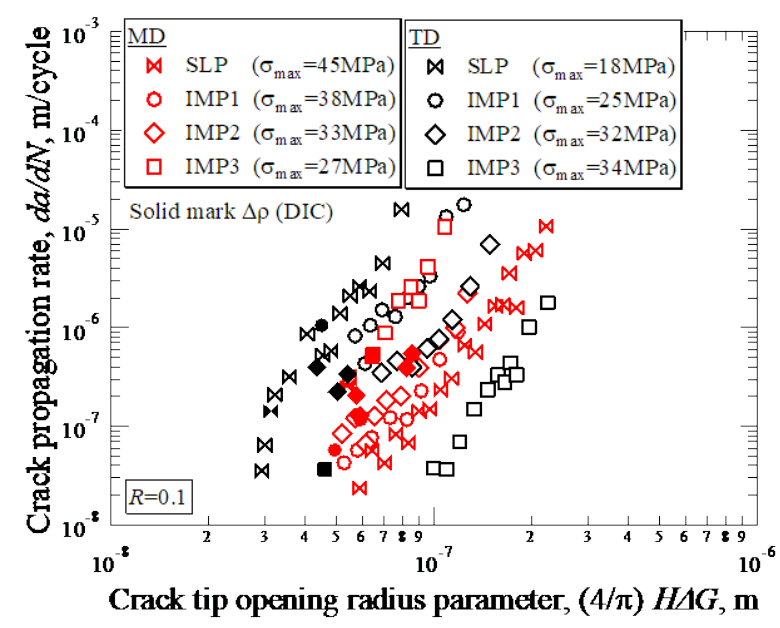

Fig. 8. Relation between crack propagation rate crack-tipopening radius.

Table 4. Effective fraction of a crack-tip-opening radius.

\begin{tabular}{c|cccc}
\hline \multirow{2}{*}{ Specimen } & \multicolumn{2}{|c}{ MD } & \multicolumn{2}{c}{ TD } \\
& $U_{\Delta \rho}$ & $\left(U_{\Delta \rho}\right)_{\text {est }}$ & $U_{\Delta \rho}$ & $\left(U_{\Delta \rho}\right)_{\text {est }}$ \\
\hline SLP & 0.14 & - & 0.96 & - \\
IMP1 & 0.35 & 0.26 & 0.49 & 0.84 \\
IMP2 & 0.63 & 0.49 & 0.36 & 0.61 \\
IMP3 & 0.84 & 0.64 & 0.10 & 0.46 \\
\hline
\end{tabular}

The relation between $d a / d N$ and a crack-tip-opening radius, $\Delta \rho(=(4 / \pi) H \Delta G)$, is shown in Fig. 8. Open marks show the converted results from $\Delta K$ using eqs. (9) and (10) and solid marks are the result of parabola approximation from measured values. Using the data from $x=0$ to $6 \times 10^{-3} \mathrm{~mm}$ in Figs. 6 and 7, the measured values are approximated by

$$
v(x)=\sqrt{\frac{x}{\alpha}}
$$

And $\Delta \rho$ is given by

$$
\Delta \rho=\frac{1}{2 \alpha}
$$

Similarly, $\Delta \rho_{\mathrm{FEM}}$ is able to obtain from the FEM analytical results in Figs. 6 and 7 and the data is equivalent to the open marks in Fig. 8.

Open marks for MD specimens come closer but the difference among TD specimens are remained similar to Fig. 5. On the other hand, solid marks for TD specimens come closer similarly to the MD specimens and the $d a / d N$ values in TD specimen is little higher than MD specimens when compared at the same value of $\Delta \rho$.

Effective fraction of a crack-tip-opening radius, $U_{\Delta \rho}$, is described as $U_{\Delta \rho}=\Delta \rho / \Delta \rho_{\mathrm{FEM}}$. From eqs.(9) and (10), the following equation is established, $U_{\Delta \rho}=U^{2}$, in which $U\left(=\Delta K_{\text {eff }} / \Delta K\right)$ is defined as an effective faction of $\Delta K$. For IMP specimens, $U_{\Delta \rho}$ is estimated from the values of SLP considering the fraction of 0 deg.(MD) and 90 deg.(TD) layer as $\left(U_{\Delta \rho}\right)_{\text {est }}$. For example, $\left(U_{\Delta \rho}\right)_{\text {est }}$ of IMP1_MD specimen is obtained by

$$
\left(U_{\Delta \rho}\right)_{\text {est }}=0.14 \times 0.86+0.96 \times 0.14=0.26
$$

Table 4 shows the values of $U_{\Delta \rho}$ and $\left(U_{\Delta \rho}\right)_{\text {est }}$. The $U_{\Delta \rho}$ value is larger than $\left(U_{\Delta \rho}\right)_{\text {est }}$ for MD specimens. And the difference increases with the increase in the thickness. It is considered that the crack length is longer in the core layer than in the shell layer and the difference becomes larger with the increase in the fraction of the core layer. Conversely, $U_{\Delta \rho}$ value is smaller than $\left(U_{\Delta \rho}\right)_{\text {est }}$ for TD specimens. It is considered that the crack length is smaller in the core layer than in the shell layer and the difference becomes larger with the increase in the fraction of the core layer.

\section{Conclusions}

The influence of plate thickness on the fatigue crack propagation behavior was studied by using centernotched specimens which were cut from injectionmolded plates of short carbon-fiber reinforced polyphenylene sulfide (PPS) at two fiber angles relative to the molding flow direction (MFD), i. e. 0 deg. (MD), $90 \mathrm{deg}$. (TD). The thickness of plates was 1,2 and $3 \mathrm{~mm}$ with three-layer structure where the fiber orientation in the shell layer is

parallel to MFD and that of the core layer is nearly perpendicular to MFD. Main results are as follows;

(1) The thickness of the shell layer does not increase even if the plate thickness increases, i. e. the fraction of the core layer increases with increase in the plate thickness.

(2) In the relation between the crack propagation rate, $d a / d N$, and stress intensity factor, $\Delta K, d a / d N$ increases with increase in thickness for MD specimen. Conversely, $d a / d N$ decreases with increase in thickness for TD specimen.

(3) The measured crack opening displacement is smaller than that analysed by FEM in shell layer plate (SLP) of MD specimen owing to the crack closure caused by the rough surface contact or the fiber bridging. On the other hand, measured values are little larger than analytical values in SLP of TD specimen. There is a possibility that the crack opening was promoted by delamination between the fiber and the matrix.

(4) The measured crack opening displacement become larger with increase in the plate thickness for MD specimens. Contrary, measured values become smaller with increase in the plate thickness for TD specimen.

(5) The crack-tip-opening radius, $\Delta \rho$, was estimated from the parabolic approximation of the crack opening displacement distribution near the crack tip. The relationships between $d a / d N$ and $\Delta \rho$ for all specimens tend to merge into a unique relationship

\section{References}

1. K. Friedrich, R. Walter, H. Voss, J. KargerKocsis, Composites, 17, pp. 205-216 (1986) 
2. M. G. Wyzgoski, G. E. Novak, Journal of Materials Science, 25, pp. 4501-4510 (1990)

3. M. G. Wyzgoski, G. E. Novak, Journal of Materials Science, 26, pp. 6314-6324 (1991)

4. W. J. Evans, D. H. Isaac, K. S. Saib, Composites Part A, 27, pp. 547-554 (1996)

5. A. Pegoretti, T. Ricco, Composite Science and Technology, 59, pp. 1055-1062 (1999)

6. A. Pegoretti, T. Ricco, Journal of Composite Materials, 34, pp. 1009-1027 (2000)

7. A. Pegoretti, T. Ricco, Composites, Part A, 33, pp. 1539-1547 (2002)

8. K. Tanaka, T, Kitano, N. Egami, Engineering Fracture Mechanics,123, pp. 44-58 (2014)

9. E. F. Rybicki, M. F. Kanninen, Engineering Fracture Mechanics, 9, pp. 931-938 (1997)

10. R. Kruger, OCASE Report No. 2002-10, NASA/CR-2002-211628 (2002)

11. G. C. Sih, P. C. Paris, G. R. Irwin, International Journal of Fracture Mechanics, 1, pp. 189-203 (1965)

12. G. C. Sih, H. Liebowitz, Fracture, Vol. 2 Mathematical Fundamentals, Academic Press, New York, pp. 67-190 (1968)

13. L. P. Pook, N. E. Frost, International Journal of Fracture Mechanics, 9, pp. 53-61(1973) 